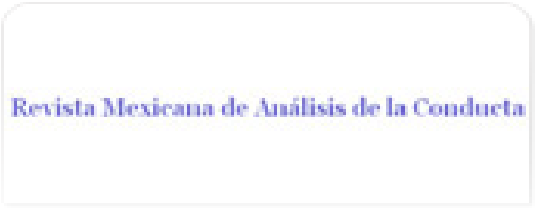

Revista Mexicana de Análisis de la Conducta ISSN: 0185-4534

editora@rmac-mx.org

Sociedad Mexicana de Análisis de la Conducta México

Bachá Méndez, Gustavo; Alonso Orozco, Ixel Discriminación de contingencias en condiciones de contrafreeloading

Revista Mexicana de Análisis de la Conducta, vol. 33, núm. 1, junio, 2007, pp. 1-17

Sociedad Mexicana de Análisis de la Conducta

Guadalajara, México

Disponible en: http://www.redalyc.org/articulo.oa?id=59333101

Cómo citar el artículo

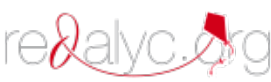

- Número completo

- Más información del artículo

Página de la revista en redalyc.org

Sistema de Información Científica

Red de Revistas Científicas de América Latina, el Caribe, España y Portugal Proyecto académico sin fines de lucro, desarrollado bajo la iniciativa de acceso abierto 


\title{
DISCRIMINACIÓN DE CONTINGENCIAS EN CONDICIONES DE CONTRAFREELOADING ${ }^{1}$
}

\author{
DISCRIMINATION OF CONTINGENCIES UNDER \\ CONDITIONS OF CONTRAFREELOADING \\ GUSTAVO BACHÁ MÉNDEZ E IXEL ALONSO OROZCO \\ FACULTAD DE PSICOLOGÍA, UNAM
}

\begin{abstract}
RESUMEN
En trabajos relacionados con el fenómeno contrafreeloading (CFL) es común utilizar un único operando durante el entrenamiento así como en las sesiones de prueba. Para corroborar la aparición de CFL en presencia de dos palancas, y con ello demostrar que más que un fenómeno anómalo es un caso particular que responde a una relación de costo-beneficio entre las diferentes fuentes disponibles, seis ratas tuvieron la oportunidad de responder a dos palancas, de las cuales sólo una de ellas proporcionaba el reforzador. Tres ratas trabajaron en un programa razón fija (RF) uno mientras las otras tres lo hicieron en RF3. En cada sesión se seleccionó de forma aleatoria una palanca para producir el reforzador, mientras la otra permanecía inactiva. Durante la línea base los reforzadores sólo podían ser obtenidos al responder a la palanca operativa mientras que en las fases de prueba además de lo anterior, se agregó, un bebedero con $80 \mathrm{ml}$ del mismo reforzador (leche). Los resultados durante las pruebas, mostraron un considerable número de respuestas a las dos palancas, con la mayoría de ellas dirigidas a la palanca operativa.
\end{abstract}

1. El experimento fue parte de los requisitos para la obtención del grado de licenciatura de la segunda autora. Porciones de este trabajo fueron presentadas en el XII Congreso Mexicano de Psicología celebrado en Guanajuato Gto; septiembre de 2004. Agradecemos a Diana Herrera y Vladimir Orduña por su valiosa revisión, y a Erika Gutiérrez y Alejandro Rangel por sus comentarios. Agradecemos también al Ing. Said Robles del Instituto de Materiales, UNAM, su apoyo en la construcción de parte del equipo.

Favor de dirigir la correspondencia a Gustavo Bachá Méndez al Laboratorio de Adaptación Animal, Facultad de Psicología, UNAM., Ave. Universidad \#3004, Colonia Copilco Universidad, CP: 04510. México, D.F. o al correo bacha@servidor.unam.mx. 
Este número de respuestas disminuyó a lo largo de las fases de prueba, lentamente para el grupo de RF1 y abruptamente para el grupo RF3. Para la discusión se consideran los patrones conductuales de exploración-explotación de recursos y de la relación costo-beneficio que ofrece cada fuente.

Palabras clave: Contra freeloading, exploración-explotación, discriminación de contingencias, palanca, ratas.

\section{SUMMARY}

In experiments related with the phenomenon contrafreeloading (CFL) it is common to use an only one operandum during the training as well as in the test sessions. To corroborate the appearance of CFL in presence of two levers, and with it to demonstrate that more than an anomalous phenomenon is a particular case that responds to a cost-benefit relationship among the available different sources, six rats had the opportunity to respond to two levers, of which only one of them provided the reinforcer. Three rats worked in a fixed ratio one (FR1) while the other ones three made it in a FR3. In each session it was selected in a random way a lever to produce the reinforcer, while the other one remained inactive. During the base line, the reinforcers could only be obtained when animal responding to the operative lever, while in the test phases besides the above-mentioned it was added a drinking trough with 80 $\mathrm{ml}$ of the same reinforcer (milk). The results during the test sessions showed a considerable number of responses to the two levers, with most of them directed to the operative lever. This number of responses diminished throughout the test phases, slowly for the group of RF1 and abruptly for the group RF3. The discussion is made in terms of patterns of exploration-exploitation of resources and of the relationship cost-benefit in each source.

Key words: Contra freeloading, exploration-exploitation, discrimination of contingencies, lever, rats

El patrón conductual conocido como "contrafreeloading" (CFL) se describe en la literatura como cierta cantidad de respuestas que un animal ejecuta en un operando para obtener un reforzador, en la presencia de una fuente que proporciona el mismo bien pero en cantidades mayores y con un costo similar o menor. Desde los primeros reportes publicados (Jensen, 1963; Neuringer, 1970), el interés se centró en tratar de explicar la cantidad de respuestas ejecutadas sobre un operando que proporcionaba pequeñas porciones de un reforzador que se encontraba presente en una fuente sin restricción para ser tomado. La mayoría de los siguientes estudios (Alferink, Crossman y Cheney, 1973; Bilbrey, Patterson y Winokur, 1973; Davidson, 1971; Enkema, Slavin, Spaeth y Neuringer, 1972; Feild, Kasper y Mitchell, 1984; Jensen, 1963; Neu- 
ringer, 1970; Osborne y Shelby, 1975; Singh y Query, 1971; Taylor, 1972) utilizaron como procedimiento básico para observar el fenómeno, una primera fase de entrenamiento para responder a un operando y con ello obtener comida por ejemplo. De manera posterior, mantener esa respuesta mediante algún programa de reforzamiento, y finalmente introducir una fuente libre del mismo alimento durante una o varias sesiones de prueba. En estas sesiones de prueba, se medía la cantidad del reforzador obtenido en cada una de las fuentes y se registró el número de respuestas al operando. Por ejemplo, Jensen (1963), entrenó a 200 ratas a oprimir una palanca en un programa de RF 1 , y una vez que la tasa de respuestas de los sujetos se estabilizó, se asignó a los animales a seis grupos. Cada grupo cumplía con un número diferente de ensayos de entrenamiento $(40,80,160,320,640$ ó 1,280) antes de enfrentar la sesión de prueba. Al inicio de la sesión de prueba, los animales eran colocados en condiciones idénticas a las del entrenamiento, pero al momento de obtener 40 reforzadores se introducía un recipiente con 250 pellas idénticas a las obtenidas por presionar la palanca. El programa de razón fija siguió funcionando pero las pellas en el recipiente podían ser consumidas libremente, estas condiciones se mantuvieron durante 40 minutos. Los resultados mostraron que las ratas con un mayor número de ensayos de entrenamiento, respondieron más para obtener comida a pesar de la presencia del recipiente con alimento no restringido. En un estudio similar, Carder y Berkowitz (1970), entrenaron a seis ratas para obtener alimento por responder sobre una palanca en un programa de RF1. Una vez que la tasa de respuestas de los animales fue estable, se introdujo la comida libre. Pero esta nueva condición se mantuvo durante dos días en un programa de RF 2 y luego se cambio a un RF 10 también durante otros dos días. Mientras el programa tenía el valor $\mathrm{RF} 1$, los seis sujetos presentaron un gran número de respuestas a la palanca para obtener el reforzador. Con el cambio a RF 2, las respuestas disminuyeron sólo en uno de los sujetos; al implementar el programa de RF10, todos los sujetos dejaron de responder a la palanca y comieron sólo de la fuente libre. Los autores concluyeron que el requerimiento del programa o esfuerzo es un factor que regula la cantidad de respuestas a la palanca en condiciones de CFL. En una variante de este procedimiento, Rutter y Nevin (1990) mantuvieron a ratas viviendo en las cajas operantes durante cuatro meses. Las cajas contenían en una extremo agua y comida de forma libre, y en el lado opuesto una palanca que al ser presionada proporcionaba pellas de $45 \mathrm{mg}$ de comida. En la primera parte del experimento, se expuso a los animales a un programa de RF 1 estando presente la comida libre. Luego, en diferentes momentos del experimento se utilizaron diferentes valores tanto de razón fija como de razón variable y una condición de extinción. Los autores reportan que los animales mantuvieron sus respuestas a los diferentes programas a pesar de la presencia del alimento libre. Sin embargo, las respuestas dismi- 
nuyeron cuando el valor de la razón fue de 43 y cuando la condición programada fue de extinción.

Una característica importante del fenómeno de CFL es que su aparición no se limita a una especie o a condiciones que involucran sólo alimentación. Aunque las pellas de comida son el reforzador más usado (Jensen, 1963; Neuringer, 1969,1970; Carder y Berkowitz, 1970) también existen trabajos en los que se empleó agua (Taylor, 1970; Bachá, 2002) o leche (Morgan, 1974). En un trabajo que estudió la conducta de peces Beta splendens se usó el reflejo del pez en un espejo como evento reforzante (Baenninger y Mattleman, 1973). En otro estudio, éste realizado con niños de edades entre 10 y 12 años (Singh y Query, 1971), la tarea consistió en presionar 10 veces un botón para obtener 5 cubos para armar. Durante las sesiones de prueba se colocó un recipiente con cubos idénticos a los que recibían los niños por presionar el botón. Los resultados mostraron que los niños continuaron respondiendo en el botón para obtener los cubos, aunque éstos estuvieran disponibles de forma libre.

Como puede observarse, el fenómeno no es exclusivo de una especie o del patrón alimenticio. La literatura confirma la presencia del CFL en diferentes condiciones, procedimientos, especies y tipos de reforzador.

Un primer punto de interés del presente trabajo esta dirigido a una variante del procedimiento utilizado en la mayoría de los estudios. En todos los trabajos reportados, el procedimiento básico incluyó el uso de un sólo operando durante el entrenamiento y en las sesiones de prueba; la única excepción conocida por los autores es el trabajo de Neuringer (1969), quien utilizó dos palancas, pero sin aclarar la razón del uso de éstas. En el presente estudio se propone el uso de dos palancas durante el entrenamiento y también durante las pruebas, para estimar si los animales responden de manera diferencial a ellos o bien si lo hacen de manera aleatoria. Para evitar algún sesgo por la posición física de las palancas se propone que en cada sesión y de forma aleatoria se elija de cuál palanca dependerá la entrega del alimento. Aparentemente en ningún trabajo previo se ha utilizado la presencia de dos operandos, esta variante permitirá mostrar si las respuestas del animal se dirigen específicamente a la palanca que provee el reforzador o bien si se distribuyen de manera similar entre las dos palancas. Una distribución semejante de respuestas a ambas palancas indicaría un efecto de activación general pero no proceso de discriminación debido a la contingencia programada. La propuesta parece importante porque en estudios anteriores, las respuestas al único operando disponible generaron explicaciones que incluyen estados inferidos poco comprobables de forma directa, por ejemplo Intrinsic Appeal (Jensen, 1963), o neofobia (Mitchell, D., Scott, W., \& Williams, K. (1973). La intención del trabajo presentado aquí es demostrar que la contingencia de reforzamiento es el factor principal que controla esas re- 
spuestas. Para lograr este objetivo, además de la presencia de dos palancas, se propone utilizarán dos valores del programa razón fija para controlar el esfuerzo requerido para obtener el reforzador programado como lo probaron Carder y Berkowitz (1970) o Inglis, Forkman y Lazarus (1997), esta manipulación permitirá confirmar los resultados de estos autores y fundamentar una interpretación de los resultados en términos de la relación costo-beneficio de ambas fuentes. En este caso se asume que para la fuente libre el costo es mínimo y el beneficio máximo, mientras que para la fuente que requiere de responder sobre una de las palancas, el beneficio será pequeño y el costo será controlado por la cantidad de respuestas requeridas por el programa de reforzamiento.

\title{
MÉTODO
}

\section{Sujetos}

Seis ratas de la cepa Wistar, con tres meses de edad al inicio del experimento y sin experiencia en procedimientos experimentales. Todos los sujetos se mantuvieron al $85 \%$ de su peso ad libitum, mediante una ración de alimento entregado al final de cada sesión. El acceso al agua siempre estuvo disponible.

\begin{abstract}
APARATOS
Dos cámaras experimentales de condicionamiento operante para ratas MED Associates Mod. ENV-007. Cada cámara se colocó dentro de una caja sonoamortiguadora de $60 \times 90 \times 80 \mathrm{~cm}$ que contenía un ventilador que funcionó como ruido blanco. La luz general fue un foco de $28 \mathrm{v}$. colocado a una distancia de $2 \mathrm{~cm}$ del techo en el panel posterior de la caja operante. En el panel frontal, a una altura de $7 \mathrm{~cm}$ desde el piso, había dos palancas con una distancia horizontal entre ellas de $17 \mathrm{~cm}$. A $7 \mathrm{~cm}$ sobre cada una de las palancas se encontraba un foco de $28 \mathrm{v}$. Entre las dos palancas, situada a una altura de $2 \mathrm{~cm}$, se encontraba una abertura cuadrada de $4 \mathrm{~cm}$ por lado que daba acceso a un bebedero que proporcionaba una gota de $0.1 \mathrm{ml}$ de leche la cual se entregaba como reforzador. En el centro del panel posterior de la caja y a una altura de $4 \mathrm{~cm}$ se encontraba una abertura rectangular de $4 \times 3 \mathrm{~cm}$ que permitía el acceso libre a una botella con $80 \mathrm{ml}$ de leche. Para ambos bebederos se utilizó leche entera ultra pasteurizada como en el trabajo de Morgan (1974). Las cajas estuvieron controladas por una interfase MED Associates (Mod. 715) y ésta se conectó a una computadora situada en un cuarto adyacente. Las sesiones experimentales se controlaron mediante
\end{abstract}


un programa elaborado en Medstate Notation y las respuestas se registraron en tiempo real.

\section{PROCEDIMIENTO}

El estudio consistió de dos fases, la primera con tres condiciones y la segunda con dos; el orden se presenta en la tabla 1. Todas las sesiones terminaron cuando los animales obtenían 50 reforzadores o después de que transcurrían 30 minutos.

Entrenamiento: En cada rata se moldeó la respuesta a la palanca para las ratas $\mathrm{X} 3, \mathrm{X} 4$ y X5 el requisito de respuesta se incrementó hasta mantener un programa de razón fija tres (RF 3 ) en cualquiera de las dos palancas. Para evitar que los animales emitieran sus respuestas sólo en una de las palancas, se alternaron los días en los que la entrega del reforzador dependía de una u otra. Para tres de las ratas $(X 3, X 4, X 5)$, se inició con la palanca derecha, alternando entre las palancas hasta completar doce sesiones Para las otras tres ratas $(\mathrm{X} 6, \mathrm{X} 7, \mathrm{X} 8)$, el ciclo se inició con la palanca izquierda. El reforzador sólo se entregaba cuando los animales cumplían el requisito de tres respuestas en la palanca elegida.

\section{FASE I}

Condición de Línea Base 1(LB1): Los animales fueron asignados a dos grupos. Para el primero de ellos (ratas X6, X7 y X8), el reforzador se obtenía cumpliendo con un programa de RF1, mientras que para el segundo grupo (ratas X3, X4 y X5), el programa utilizado fue RF3. Antes de iniciar cada sesión, el programa de control elegía al azar la palanca asociada con el reforzador, la cual se identificará como la palanca operativa $(P O)$. Mientras que, la otra palanca, identificada como la palanca no operativa (PNO), no producía el reforzador ni los otros estímulos asociados con la recompensa. Si los animales respondían a la $\mathrm{PO}$, se apagaba la luz general y las luces sobre las palancas, sonaba un tono durante un segundo y se entregaba una gota de $0.1 \mathrm{ml}$ de leche a la cual llamaremos fuente programada. Las condiciones de esta primera fase se mantuvieron durante 30 sesiones.

Condición prueba 1: A las condiciones de la Línea Base 1 se agregó el acceso a un bebedero. Al inicio de cada sesión se abría la puerta del panel posterior de la caja operante para dar acceso al bebedero que contenía 80 $\mathrm{ml}$ de leche. Este bebedero se nombrará como la fuente no restringida. Al término de cada sesión se retiraba el bebedero libre, se vaciaba la leche restante en una pipeta graduada en mililitros para calcular la cantidad bebida por 
cada rata. Estas condiciones se mantuvieron durante 30 sesiones.

Condición de Línea Base 2 (LB2): Se repitieron las condiciones de la Línea Base 1 durante diez sesiones.

\section{FASE II}

Condición de Línea Base 3-Prueba 2: Esta condición representa una prueba adicional del control que ejercen las condiciones programadas para la sesión. Al inicio de cada sesión se decidía de forma aleatoria, si estaría presente la fuente no restringida (sesiones de Prueba) o no lo estaría (sesiones de Línea Base). Como resultado de este procedimiento, la distribución de sesiones con las condiciones de línea base y de prueba, fue diferente para cada sujeto. Esto permite realizar una comparación con los resultados del otro conjunto de sesiones de prueba. Esta fase concluyó cuando los animales acumularon 10 sesiones en las que existía acceso a la fuente no restringida; por lo tanto el número de sesiones fue variable para cada sujeto.

\begin{tabular}{cccc|c} 
& \multicolumn{3}{c|}{ Fase I } & Fase II \\
\cline { 2 - 5 } SUJETOS & LB 1 & Prueba 1 & LB 2 & Prueba 2* \\
\hline X 6 & & & & \\
X 7 & RF 1 & RF 1 + FNR & RF 1 & RF 1 + FNR \\
X 8 & & & & \\
\hline X 3 & & & & \\
X 4 & RF 3 & RF 3 + FNR & RF 3 & RF 3 + FNR \\
X5 & & & & \\
\hline
\end{tabular}

Tabla 1: Diseño utilizado mostrando cada una de las Fases experimentales.

Nota: $L B=$ Línea Base, $R F=$ razón fija, $F N R=$ Fuente no restringida.

*Las sesiones en esta Fase se alternaron, entre líneas base y pruebas.

\section{RESULTADOS}

Para comparar la ejecución de los sujetos en las diferentes fases y estimar la diferencia entre los grupos RF1 y RF3, se calcularon el promedio y el error estándar de la frecuencia de respuestas a la palanca operativa (PO) y a la 
palanca no operativa (PNO) en bloques de cinco días. De forma adicional se presentan los porcentajes de respuesta dirigidas a cada una de las palancas, durante las líneas base así como durante las fases de prueba para ambos grupos.

La figura 1 presenta para cada sujeto del grupo RF1, el promedio de respuestas en la palanca operativa (círculos obscuros) y las respuestas en la palanca no operativa (círculos blancos) en bloques de cinco sesiones. En las gráficas de la izquierda se presentan los datos de la Fase I, que incluye las condiciones de LB1, la Prueba 1 y la LB 2. Las gráficas de la derecha muestran los datos de la Fase II, la cual se dividió agrupando las sesiones tipo línea base y aquellas en las que estuvo presente la fuente no restringida. Durante la Línea Base 1 se observa que para los tres sujetos, las respuestas en la $\mathrm{PO}$ se mantuvieron en el valor 50 que representa el número máximo de reforzadores que podía obtenerse. Las respuestas en la PNO mantienen una frecuencia variable pero siempre menor a las respuestas a la $\mathrm{PO}$. En la siguiente fase, al agregar la fuente no restringida, se advierte que el total de respuestas declina para los tres sujetos, disminuyendo principalmente en las respuestas a la palanca operativa. Los valores al final de la fase fueron: 22 para el sujeto X6, 25 para el X8, y cinco para el sujeto X7. Las respuestas en la PNO mantuvieron un nivel semejante al observado durante la línea base. Al reinstalar las condiciones de línea base todos los animales incrementaron sus respuestas en la PO obteniendo todos los reforzadores y manteniendo la frecuencia de respuestas en la PNO por debajo de 12.

En el panel de gráficas a la derecha de la figura 1 se muestra la Fase II con los resultados separados para las sesiones de línea base y aquellas de prueba. Es posible advertir que para los tres sujetos, las respuestas en la PO durante las sesiones de línea base siempre fueron 50, consiguiendo todos los reforzadores, mientras que las respuestas a la PNO se mantuvieron en valores menores a 12 por bloques de 5 sesiones. En las sesiones con acceso a la fuente no restringida, los tres animales disminuyeron sus respuestas en la PO. Las ratas X6 y X8 mantuvieron durante los dos bloques una diferencia a favor de la $\mathrm{PO}$, mientras que para la rata $\mathrm{X} 7$ las respuestas fueron muy similares en ambos bloques. 

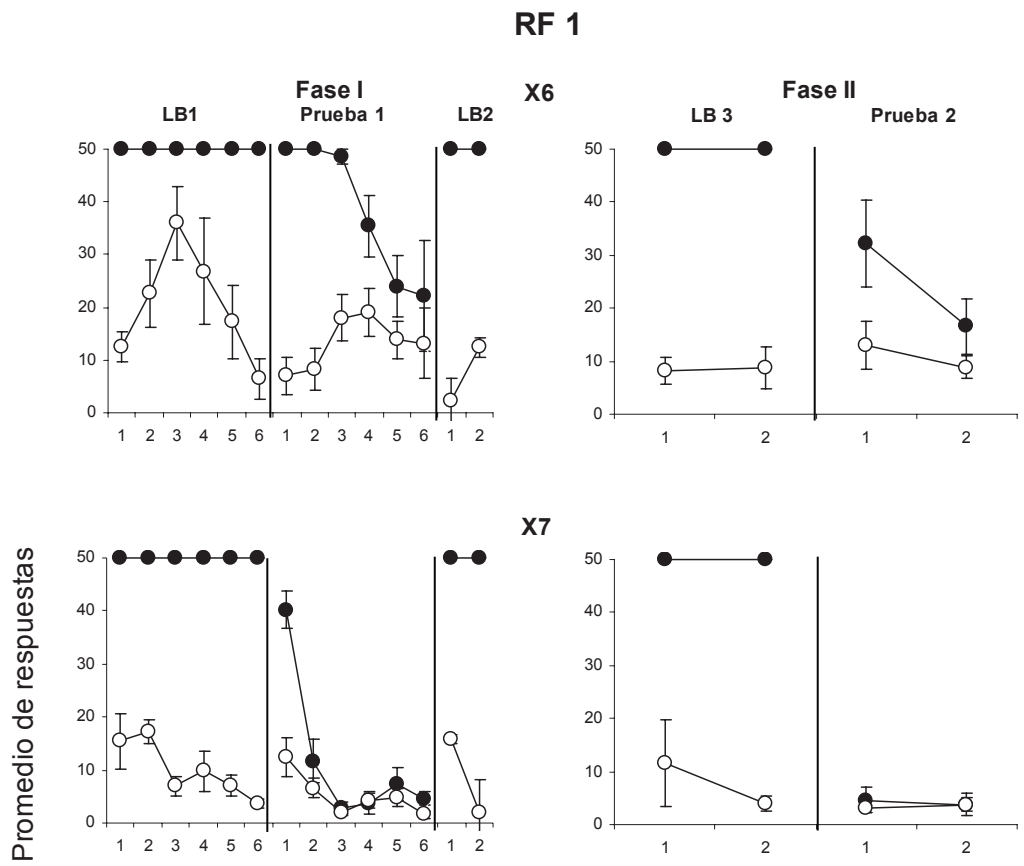

X7
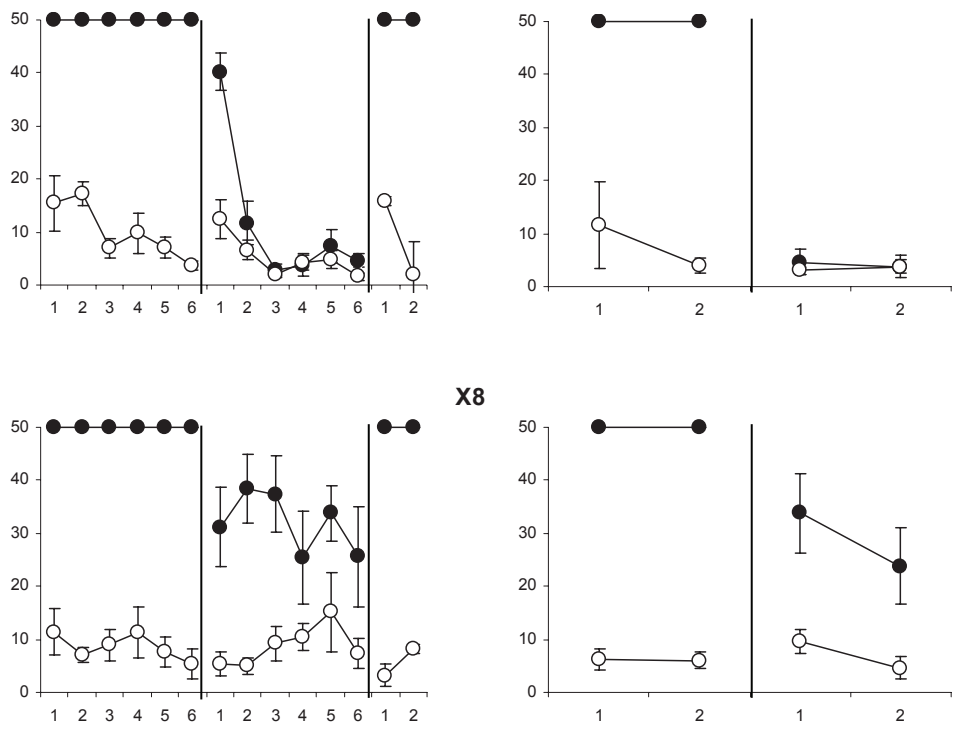

$x 8$

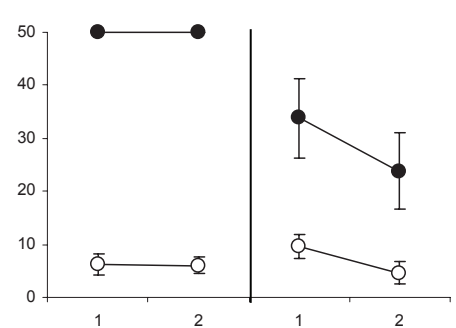

Bloques de 5 sesiones

Figura 1: Comparación de las frecuencias de respuestas a cada una de las palancas (grupo RF1). En el horizontal se presentan bloques de cinco sesiones y en el eje vertical la frecuencia de respuestas: Los círculos obscuros muestran las respuestas en la palanca operativa y los claros las respuestas en la palanca no operativa. Las gráficas de la izquierda muestran los datos de la Fase I con sus tres condiciones y las gráficas de la derecha la Fase II con sus dos condiciones. 
En la figura 2 se presentan las gráficas de los sujetos que estuvieron en el grupo RF3. Al igual que para el grupo RF1, el panel izquierdo muestra los datos de la Fase I, en la cual se observa las condiciones de LB1, la prueba 1 y la LB 2 , mientras que las gráficas de la derecha muestran los datos de la Fase 2. El eje vertical tiene un máximo de 150 debido a que los sujetos trabajaron bajo un programa de RF3. Durante la LB1, las respuestas de los tres sujetos en la PO fueron 150 (con lo que obtenían todos los reforzadores disponibles), en tanto que las respuestas en la PNO fueron disminuyendo a lo largo de la condición terminando por debajo de 10 respuestas en promedio. En la Prueba 1 se observa que la cantidad de respuestas disminuye para ambas palancas. Esta disminución ocurrió desde el primer bloque de sesiones para los sujetos X3 y X5 mientras que para el sujeto X4 el decremento se inicia a partir del cuarto bloque. Al reinstalar las condiciones de LB2, todos los animales incrementaron las respuestas en la PO obteniendo todos los reforzadores, y las respuestas a la PNO se mantienen por debajo de las 20 respuestas por sesión. En el panel de la derecha se muestran los datos de sesiones de tipo línea base y a un lado aquellas semejantes a la prueba anterior. Durante la condición sin acceso libre a la leche los tres sujetos obtuvieron todos los reforzadores, esto es respondieron 150 veces en la PO. Las respuestas en la PNO se mantuvieron en todos las ratas en un nivel promedio no mayor a 10. Durante las sesiones con acceso a la fuente no restringida, las respuestas a ambas palancas disminuyeron en ambos bloques para todos los sujetos.

En la tabla 2 se presentan el promedio de respuestas totales en cada condición y el porcentaje promedio de respuesta a cada una de las palancas. Los datos del grupo RF1 se encuentran en el panel superior y en el inferior los del grupo RF3. La columna con el promedio de respuestas totales muestra que los animales en cada grupo mantienen una cantidad similar de respuestas durante las líneas base y que esta cantidad disminuye durante las condiciones de prueba PR1 y PR2. En todos los casos la cantidad de respuestas a las palancas fue mayor durante la PR1 en comparación con la PR2. En la cuarta columna se muestra que el mayor porcentaje de estas respuestas se dirigió a la PO, con la excepción del sujeto X7 (grupo RF1) y del sujeto X3 (grupo RF3) ambos casos durante la segunda prueba.

En el panel superior de la tabla 2, se aprecia que para el grupo RF1, las respuestas a la $\mathrm{PO}$ durante las tres líneas base, se ubican por encima del $80 \%$ del total de las respuestas excepto en el caso del sujeto X6 en la LB1 que asignó el $75 \%$ de las respuestas totales la palanca operativa. Durante la primera prueba (PR1) las repuestas a la PO diminuyen su porcentaje pero se mantienen en un rango de 61 a $76 \%$. Durante la segunda prueba (PR2) dos de los sujeto mantienen una buena cantidad de respuestas totales y un porcentaje semejantes a la $\mathrm{PO}$. La excepción es el sujeto X7 que mantuvo muy pocas respuestas totales distribuyó el $43 \%$ de ellas a la PO. 
RF 3

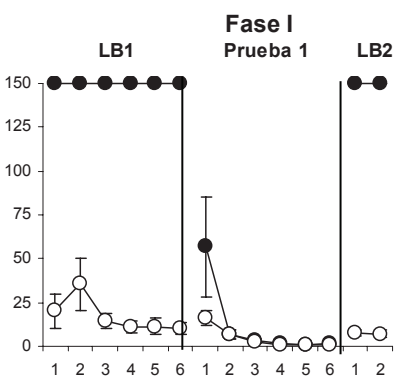

$\mathrm{X} 3$
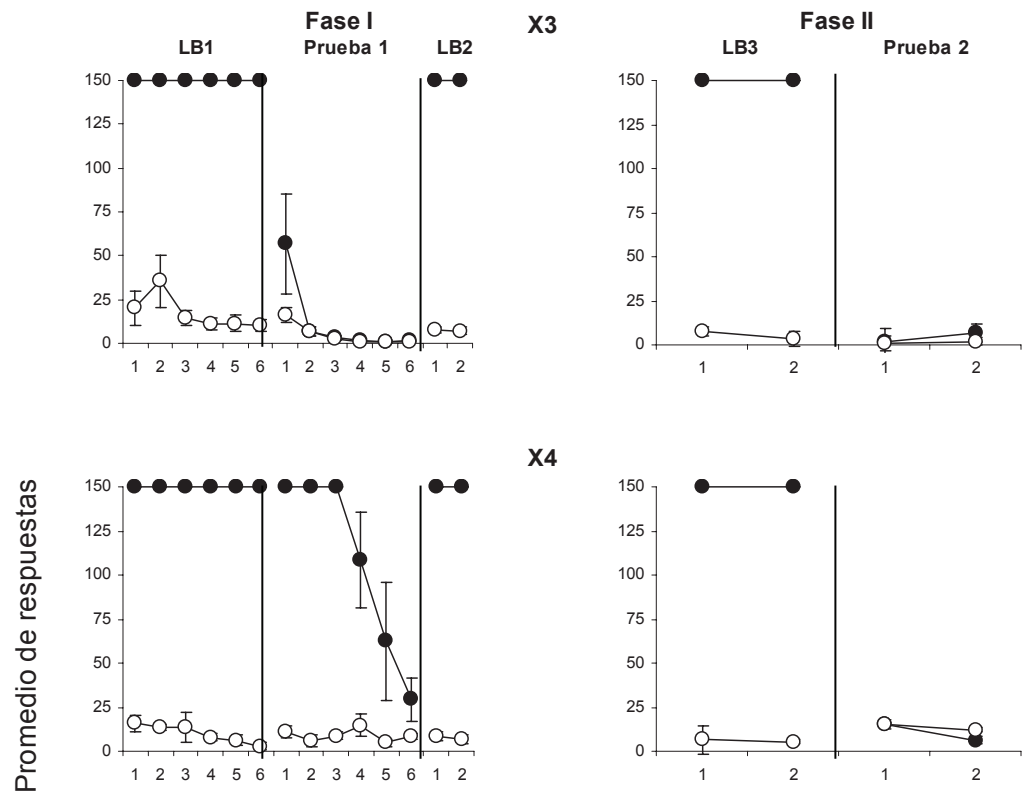

X4
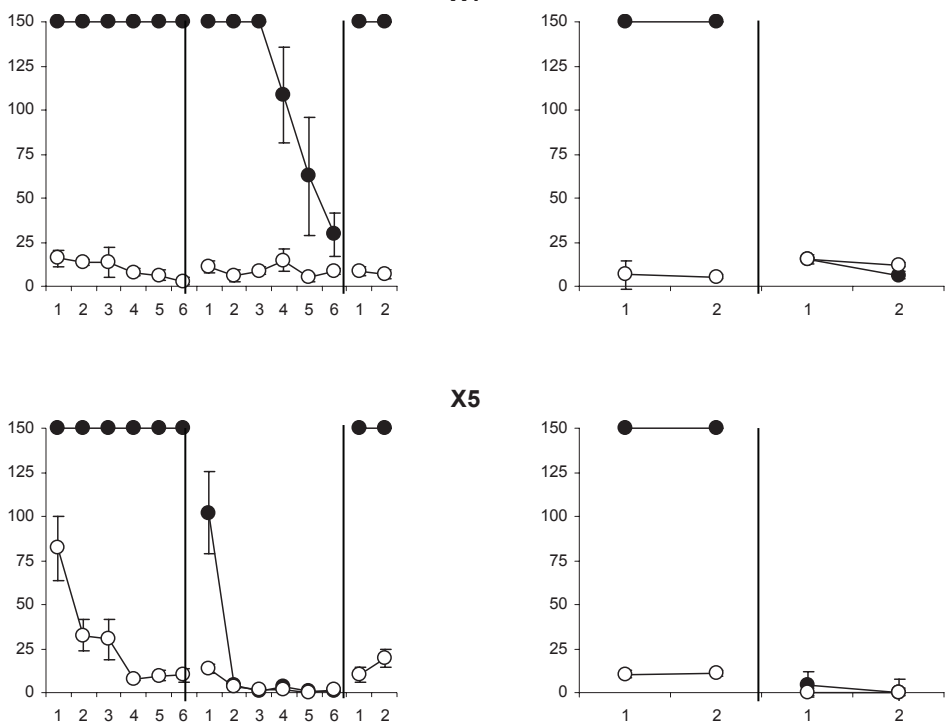

X5

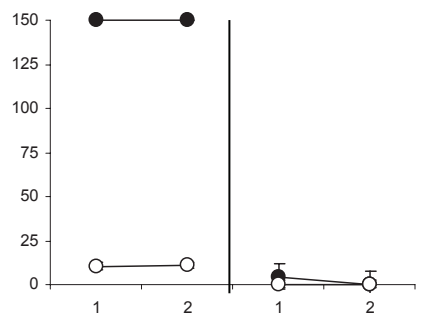

Bloques de 5 sesiones

Figura 2: Comparación de las frecuencias de respuestas a cada una de las palancas (grupo RF3). En el horizontal se presentan bloques de cinco sesiones y en el eje vertical la frecuencia de respuestas: Los círculos obscuros muestran las respuestas en la palanca operativa y los claros las respuestas en la palanca no operativa. Las gráficas de la izquierda muestran los datos de la Fase I con sus tres condiciones y las gráficas de la derecha la Fase II con sus dos condiciones. 


\begin{tabular}{|c|c|c|c|c|}
\hline \multirow{4}{*}{ Sujeto } & \multirow{4}{*}{ Condiciones } & \multirow{2}{*}{\multicolumn{3}{|c|}{ Grupo RF1 }} \\
\hline & & & & \\
\hline & & \multirow[b]{2}{*}{ Promedio respuestas } & \multicolumn{2}{|c|}{ Porcentajes Promedio } \\
\hline & & & PO & PNO \\
\hline \multirow{5}{*}{ X6 } & LB 1 & 70 & 75 & 25 \\
\hline & LB 2 & 58 & 88 & 12 \\
\hline & LB 3 & 58 & 86 & 14 \\
\hline & PR 1 & 52 & 72 & 28 \\
\hline & PR 2 & 35 & 64 & 36 \\
\hline \multirow{5}{*}{$\mathbf{X} 7$} & LB 1 & 60 & 85 & 15 \\
\hline & LB 2 & 59 & 88 & 12 \\
\hline & LB 3 & 58 & 89 & 11 \\
\hline & PR 1 & 17 & 61 & 39 \\
\hline & PR 2 & 8 & 43 & 57 \\
\hline \multirow{5}{*}{ X8 } & LB 1 & 59 & 87 & 13 \\
\hline & LB 2 & 56 & 89 & 11 \\
\hline & LB 3 & 56 & 89 & 11 \\
\hline & PR 1 & 41 & 76 & 24 \\
\hline & PR 2 & 36 & 78 & 22 \\
\hline & & \multicolumn{3}{|c|}{ Grupo RF3 } \\
\hline & & & \multicolumn{2}{|c|}{ Porcentajes Promedio } \\
\hline Sujeto & Condiciones & Promedio respuestas & PO & PNO \\
\hline \multirow{5}{*}{$\mathbf{X 3}$} & LB 1 & 167 & 91 & 9 \\
\hline & LB 2 & 157 & 95 & 5 \\
\hline & LB 3 & 156 & 97 & 3 \\
\hline & PR 1 & 19 & 77 & 23 \\
\hline & PR 2 & 5 & 75 & 25 \\
\hline \multirow{5}{*}{ X4 } & LB 1 & 160 & 94 & 6 \\
\hline & LB 2 & 158 & 95 & 5 \\
\hline & LB 3 & 156 & 96 & 4 \\
\hline & PR 1 & 117 & 83 & 17 \\
\hline & PR 2 & 25 & 44 & 56 \\
\hline \multirow{5}{*}{ X5 } & LB 1 & 179 & 86 & 14 \\
\hline & LB 2 & 163 & 93 & 7 \\
\hline & LB 3 & 161 & 94 & 6 \\
\hline & PR 1 & 23 & 86 & 14 \\
\hline & PR 2 & 3 & 89 & 11 \\
\hline
\end{tabular}

Tabla 2: Promedio de respuestas totales durante cada una de las fases y promedio de porcentajes de respuesta a cada una de las palancas. En el panel superior se presentan resultados de los sujetos que estuvieron en el grupo RF1 y en el panel inferior, los valores de los sujetos que formaron el grupo que trabajó con un programa RF3.

Nota: LB1 = Línea Base 1; PR1 = prueba 1; PR2 = prueba 2. 
En el panel inferior se presentan los datos de los sujetos del grupo RF3. Las respuestas totales durante las líneas base fueron muy semejantes en todos los animales. El porcentaje dirigido a la PO durante las líneas base se ubicó por encima del 90\%, excepto el sujeto X5 que en la LB1 respondió el $86 \%$ de las respuestas totales en la PO, pero que posteriormente lo incrementó. Para este grupo, las respuestas totales disminuyeron mucho más que en el grupo RF1 durante la primera prueba (PR1). El único animal que mantuvo una cantidad considerable de respuestas fue X4. En la PR2 la cantidad total de respuesta para los tres animales fue muy pequeña en relación con su línea base. Sin embargo, los porcentajes dirigidos a la PO fueron mayores que en el grupo RF1. Con la excepción del sujeto X4 cuyo porcentaje fue 44 durante la segunda prueba, todos los otros porcentajes son en promedio superiores a los del grupo RF1.

\section{DISCUSIÓN}

El propósito del trabajo fue mostrar que la contingencia de reforzamiento es la principal variable controladora de las respuestas en la condición de CFL. Los resultados confirman que el fenómeno de CFL se presentó en ambos grupos, pero que además las respuestas fueron dirigidas en su mayoría a la PO a pesar de que en cada sesión se elegía de manera aleatoria cuál de las dos palancas presentes estaría asociada con el reforzador. Asimismo, la diferencia en el valor del programa, propició una diferencia en el porcentaje de respuestas a la palanca operativa durante las pruebas. Este último dato confirma lo reportado por Carder y Berkowitz (1970), Inglis, Forkman y Lazarus, (1997) y Rutter y Nevin, (1990) en términos de que el valor del programa influye en la cantidad de respuestas emitidas en presencia de la fuente libre. Sin embargo, a diferencia de trabajos en los que existe un solo operando, con la presencia de dos palancas se demostró que tanto durante las líneas base como en las pruebas, la condición de contingencia controló que el porcentaje de respuestas dirigidas a la PO fuera mayor.

Los datos de ambos grupos muestran que durante las condiciones de prueba los animales disminuyen su número de respuestas a partir de los primeros bloques, de forma gradual para el grupo RF1 y de manera abrupta para el grupo RF3. Esta disminución se presentó en todos los sujetos, lo que sugiere que el CFL (mantener respuestas al operando en presencia de una fuente no restringida del reforzador) disminuye con el paso de las sesiones experimentales. La mayoría de los autores (Jensen, 1963; Carder y Berkowitz, 1970; Neuringer, 1970; Osborne y Shelby, 1975 y Forkman, 1996), reportan sólo las primeras 5 a 10 sesiones, sin mencionar una disminución como la observada en este trabajo. En el presente estudio, al conservar las 
condiciones de prueba durante 30 sesiones, se observó que la cantidad de respuestas diminuye a valores menores a 10 respuestas por sesión. Aunque a este respecto es probable que el resultado sea diferente en el caso de que los animales vivan todo el tiempo en la cámara experimental y la economía fuera cerrada (Rutter, S. y Nevin, J. 1990 Hursh, 1984).

Los resultados de la distribución de respuestas a las palancas confirman que, a pesar de la elección aleatoria de la palanca que proporcionaría el reforzador, la preferencia por la PO se mantuvo en un rango de 75 hasta $97 \%$ durante las líneas base. Y durante las condiciones de prueba, 10 de los 12 porcentajes se situaron en un rango de 61 a $89 \%$ Estos porcentajes representan un índice de preferencia por la PO, que parece confirmar que el control de la contingencia de reforzamiento ejercido durante la línea base no se pierde durante las sesiones de prueba.

La medición de la cantidad de leche tomada de cada una de las fuentes (dato no reportado) indica que todos los animales obtenían $5 \mathrm{ml}$ de leche durante la línea base, esta cantidad se incrementó a un rango entre los 25 y 30 $\mathrm{ml}$ durante las fases de prueba. La leche obtenida de la fuente no restringida siempre representó un porcentaje superior al $75 \%$ llegando a ser del $95 \%$. El dato es importante porque representa otro indicio de que a pesar de representar una proporción pequeña del total ingerido, el reforzador programado mantiene algún valor.

Por mucho tiempo, la explicación del CFL ha sido un tema controvertido. Por ejemplo, Jensen (1963) en lo que puede considerarse uno de los primeros trabajos, propuso que lo que controla la respuesta de los animales en presencia de una fuente libre es un interés intrínseco (intrinsic appeal), es decir que a las ratas les "gusta" responder a la palanca. Este tipo de explicaciones parecen implicar que el valor del reforzador dependiente de la respuesta tiene muy poco valor de incentivo. Esta visión en la que un factor diferente a la contingencia programada es la responsable de las respuestas de los animales al operando ha dado lugar a una serie de explicaciones parciales (algunas importantes) de la conducta observada. Entre las explicaciones se pueden mencionar, que las respuestas al operando se mantienen por el cambio de estímulos asociados a la entrega del reforzador (Wallace, Osborne, Norborg y Fantino, 1973; Osborne y Shelby, 1975) lo cuál es probable que sea cierto pero no suficiente. Otra posibilidad es que las respuestas se mantienen por "intrinsic appeal" como lo planteo Jensen (1963), que luego Mahoney y Bandura (1972) interpretaron como un auto-reforzamiento; o bien, que se sigue respondiendo en presencia de la fuente no restringida debido a la neofobia planteada por Mitchell, Scott y Williams (1973) quienes manipularon la familiaridad y la novedad de los recipientes que contenían tanto la comida que ganaban los animales por sus respuestas como la comida libre. Una excelente 
compilación de las explicaciones que se han desarrollado se puede encontrar en Osborne (1977) y también en el trabajo de Inglis et al. (1997).

En la presente investigación, los resultados se interpretan principalmente como el efecto de la contingencia programada para las respuestas a las palancas y la presencia o ausencia de la fuente no restringida. Es posible ver en las condiciones impuestas un compromiso entre la exploración o muestreo de fuentes y su explotación (Staddon, 1983). En la línea base, la conducta de los sujetos era dirigida a discriminar de cuál palanca depende la entrega del reforzador. Durante las fases de prueba, la presencia de la fuente libre, representa la oportunidad de explorar y explotar dos fuentes, una que con un costo muy bajo proporciona una cantidad mayor de reforzador en comparación con otra que además de ofrecer un beneficio menor requiere de encontrar cuál palanca proporciona esa pequeña cantidad. El uso, en este trabajo, de dos valores del programa asociado a las respuestas en las palancas, representó un costo bajo (RF1) o bien uno más elevado (RF3). En estas condiciones la conducta de exploración de ambas fuentes y la decisión, con base en el costo y el beneficio obtenido, de la forma de distribuir su conducta no requiere de explicaciones diferentes a las derivadas del análisis de las contingencias impuestas a los animales. Esta interpretación de los resultados coincide con el trabajo de Rachlin y Baum (1972), sobre la importancia de una fuente alterna de reforzamiento y la forma en que los sujetos distribuyen su conducta ante dos o más fuentes de reforzamiento. Este problema esta planteado de forma clara en el trabajo de Herrnstein (1970) sobre la llamada ley de igualación. Los resultados obtenidos demuestran que el reforzador conseguido mediante la respuesta a la PO mantiene su valor, aún en presencia de la fuente no restringida. $Y$ que estas respuestas son sensibles al costo impuesto por el programa de razón, siguiendo probablemente reglas como igualación (Herrnstein, 1970) o maximización (Rachlin, H., Battalio, R., Kagel, J. y Greenl, 1981).

En una serie de reportes, autores como Forkman (1996), Inglis y Ferguson (1986) e Inglis y Shepherd, (1994), han propuesto que un factor importante para la aparición del CFL es la "adquisición de información" (entendiendo información como reducción de incertidumbre). Esta aproximación no representa un problema diferente al planteado, ya que lo que parece implicar, es que la obtención de información derivada de la regla de contingencia entre la conducta y sus consecuencias es la responsable de su mantenimiento bajo condiciones de CFL. En otro terreno afín, para autores como Kacelnik (1987) y Krebs, Kacelnick y Taylor (1978) que han intentado integrar el CFL al área de forrajeo bajo incertidumbre, tampoco sería difícil aceptar que el factor importante es el de la contingencia de reforzamiento. Algunas preparaciones propician la exploración o búsqueda de fuentes alternas, mientras que otras minimizan ese patrón debido por ejemplo a una explotación prolongada 
de una de ellas. La conducta de los organismos es sensible a la relación existente entre el costo o esfuerzo exigido para la obtención de un bien y el beneficio (cantidad - calidad) que representa la obtención del mismo. En el presente estudio los animales demostraron ser sensibles a las condiciones en las que se presentaron dos fuentes del mismo reforzador. Para concluir, es necesario reconocer que los resultados del presente trabajo no pueden descartar de manera directa los otros factores propuestos para explicar la presencia del CFL. Pero los resultados si muestran que este patrón conductual no debería ser tratado como una categoría anómala, ya que es posible describirlo y entenderlo a partir de las reglas de contingencia impuestas y de las relaciones de costo - beneficio proporcionadas por las fuentes presentes en las condiciones en las que fue estudiado.

\section{REFERENCIAS}

Alferink, L., Crossman, E. y Cheney. (1973). Control of responding by a conditioned reinforcer in the presence of free food. Animal Learning and Behavior, 1, 38-40.

Bachá, G. (2002). Adquisición de una respuesta operante en presencia de una fuente alterna: Contrafreeloading. Revista Mexicana de Análisis de la Conducta, 28, 91104.

Baenninger, R., \& Mattleman, R. (1973). Visual reinforcement: Operant acquisition in the presence of a free mirror. Animal Learning and Behavior, 1(4), 302-306.

Bean, D., Mason, G. y Bateson, M. (1999). Contrafreeloading in starlings: testing the information hypothesis. Behaviour, 136, 1267-1282.

Bilbrey, J., Patterson, D. y Winokur, S. (1973). Maintenance and autoshaping of keypecking in undeprived pigeons. Bulletin of the Psychonomic Society, 3(6A), 394396.

Carder, B. y Berkowitz, K. (1970). Rats' preference for earned in comparison with free food. Science, 167, 1273-1274.

Davidson, A. (1971). Factors affecting keypress responding by rats in the presence of free food. Psychonomic Science, 24(3), 135-137.

Enkema, S., Slavin, R., Spaeth, C. y Neuringer, A. (1972). Extinction in the presence of free food. Psychonomic Science, 25(5), 267-269.

Feild, C., Kasper, S. y Mitchell, D. (1984). Effort and contrafreeloading. Bulletin of the Psychonomic Society, 22(2), 147-150.

Forkman, B. (1996). The foraging behaviour of mongolian gerbils: A behavioural need or need to know? Behaviour, 133, 129-143.

Herrnstein, R. J. (1970). On the law of effect. Journal of the Experimental Analysis of behavior, 13, 243-266.

Hursh, S. (1984). Behavioral economics. Journal of Experimental Analysis of Behavior, 42(3), 435-452.

Inglis, I., \& Ferguson, N. (1986). Starlings search for food rather then eat freely-available, identical food. Animal Behaviour, 34(2), 614-617. 
Inglis, I., Forkman, B. y Lazarus, J. (1997). Free food or earned food? A review and fuzzy model of contrafreeloading. Animal Behaviour, 53, 1171-1791.

Inglis, I., \& Shepherd, D. (1994). Rats work for food they then reject: Support for the Information-primacy Approach to Learned Industriousness. Ethology, 98, 154-164.

Jensen, G. (1963). Preference for bar pressing over freeloading as a function of number of rewarded presses. Journal of Experimental Psychology, 65(5), 451-454.

Kacelnik, A. (1987). Information primacy or preference for familiar foraging techniques? a critique of Inglis \& Forkman. Animal Behaviour, 35(3), 925-926.

Krebs, J. (1978). Optimal Foraging: Decision Rules for predators. In J. Krebs \& N. Davies (Eds.), Behavioral Ecology an evolutionary approach.

Mahoney, M., \& Bandura, A. (1972). Self-Reinforcement in Pigeons. Learning and motivation, 3, 293-303.

Mitchell, D., Scott, W., \& Williams, K. (1973). Container neophobia and rat's preference for earned food. Behavioral Biology, 9, 613-624.

Morgan, M. (1974). Do rats like to work for their food? Learning and Motivation, 5(352368).

Neuringer, A. (1969). Animals respond for food in the presence of free food. Science, 166, 399-401.

Neuringer, A. (1970). Many respond per food reward with free food present. Science, 196, 503-504.

Osborne, S. y Shelby, M. (1975). Stimulus change as a factor in response maintenance with free food available. Journal of Experimental Analysis of Behavior, 24, 17-21.

Osborne, S. R. (1977). The free food (contrafreeloading) phenomenon: A review and analysis. Animal Learning Behavior, 5,221-235

Rachlin, H. C., Battalio, R., Kagel, J. y Greenl. (1981). Maximization theory in behavioral psychology. Behavioral and Brain Sciences, 4, 371-417.

Rachlin, H. y Baum, W. (1972). Effects of alternative reinforcement: does the source matter? Journal of Experimental Analysis of Behavior, 18(2), 231-241.

Rutter, S. y Nevin, J. (1990). Long-term contrafreeloading. Bulletin of the Psychonomic Society, 28(6), 556-558.

Shepherdson, D., Carlstead, K., Mellen, J. y Seidensticker, J. (1993). The influence of food presentation on the behavior of small cats in confined environments. Zoo Biology, 12, 203-216.

Singh, D.y Query, W. (1971). Preference for work over "freeloading" in children. Psychonomic Science, 24(2), 77-79.

Staddon, J. E. R. (1983). Learning II: The guidance of action. Adaptative behavior and learning (pp. 451-452).

Taylor, G. (1972). A limitation of the contrafreeloading phenomenon. Psychonomic Science, 29(3), 173-174.

Wallace, R. F., Osborne, S., Norborg, J., y Fantino, E. (1973). Stimulus change contemporaneous with food presentation maintains responding in the presence of free food. Science, 182, 1038-1039. 